\title{
AUTOEFICACIA, PERCEPCIÓN DE BARRERAS Y BENEFICIOS DE LA ACTIVIDAD FÍSICA EN ESTUDIANTES UNIVERSITARIOS COSTARRICENSES
}

\section{PHYSICAL ACTIVITY SELF-EFFICACY, THE PERCEPTION OF BARRIERS AND BENEFITS IN COSTA RICAN UNIVERSITY STUDENTS}

\author{
Angélica Ortega Muñoz ${ }^{1}$, Sergio Fumero Pérez ${ }^{2}$ y Ana Laura Solano López ${ }^{3}$ \\ angelik.m11@gmail.com; sefupe@gmail.com; analaura.solanolopez@ucr.ac.cr \\ ${ }^{1}$ Hospital Rafael Ángel Calderón Guardia, San José, Costa Rica \\ ${ }^{2}$ Ministerio de Salud, San José, Costa Rica \\ ${ }^{3}$ Universidad de Costa Rica, San José, Costa Rica
}

Envio Original: 2020-11-17 Reenviado: 2021-03-05 Aceptado: 2021-07-12

Publicado: 2021-08-17

Doi: https://doi.org/10.15517/pensarmov.v19i2.44669

\begin{abstract}
RESUMEN
Según la Teoría de Promoción de la Salud aspectos como autoeficacia, percepción de beneficios y barreras podrían estar influyendo en la actividad física. En Costa Rica se ha investigado poco este fenómeno en estudiantes universitarios de enfermería. Por lo que el objetivo del estudio fue determinar la relación entre autoeficacia y percepción beneficios y barreras de la actividad física en estudiantes de Enfermería de la Universidad de Costa Rica. Esta investigación es un estudio de caso grupal, correlacional descriptivo. La población estuvo conformada por 42 estudiantes de segundo año de Enfermería. Los instrumentos utilizados fueron la Escala de Beneficios/Barreras para el ejercicio, y la Escala de Autoeficacia para la actividad física. El análisis de datos se realizó mediante estadística descriptiva y la correlación de Pearson. Dentro de los resultados resalta que caminar obtuvo mayor autoeficacia seguido de actividades de la vida diaria y por último el ejercicio físico programado. El estudiantado identifica más los beneficios de la actividad física que las barreras. Existe una asociación entre la autoeficacia total para la actividad física y la percepción de beneficios del ejercicio y la autoeficacia para el ejercicio programado y las barreras del ejercicio. Se debe trabajar en potenciar los beneficios de la actividad física, así como en crear intervenciones que ayuden a la población estudiantil de enfermería a superar las barreras por
\end{abstract}


medio del fortalecimiento de la autoeficacia para la actividad física independientemente de su intensidad.

Palabras clave: autoeficacia, actividad física, estudiantes, enfermería.

\begin{abstract}
According to the Health Promotion Theory, aspects such as self-efficacy, the perception of benefits and barriers could be influencing physical activity. In Costa Rica little research has been done on this phenomenon in university students. Therefore, the study aim was to determine the relationship between self-efficacy and the perception of benefits and barriers of physical activity in nursing students. The research is a descriptive correlational group case study. The population consisted of 42 second-year nursing students. Instruments used were the Scale of Benefits / Barriers for Exercise, and the Self-efficacy Scale for Physical Activity. Data analysis was performed using descriptive statistics and Pearson's correlation. Among the results stands out that walking obtained greater self-efficacy followed by activities of daily living and finally programmed exercise Students identify more the benefits of physical activity than barriers. There is an association between total self-efficacy for physical activity and perceived benefits of exercise and self-efficacy for programmed exercise and exercise barriers. Work should be done to enhance the benefits of physical activity, as well as to create interventions that help nursing students overcome barriers by strengthening their self-efficacy for physical activity regardless of its intensity.
\end{abstract}

Keywords: self-efficacy, physical activity, students, nursing

\title{
INTRODUCCIÓN
}

La actividad física (AF) es un elemento fundamental de la salud integral de las personas. Uno de cuatro adultos en el mundo no realiza suficiente AF para obtener beneficios para la salud, posicionando la inactividad física como el cuarto factor de riesgo más importante asociado a la mortalidad y a la alteración en indicadores de salud mental (Organización Mundial de la Salud [OMS], 2018; Cruz-Castruita, González, Raimundi, y Enríquez-Reyna, 2017; Rodríguez-Romo, Barriopedro, Salazar, y Garrido-Muñoz, 2015).

Esta problemática podría tener especial impacto en población estudiantil universitaria, ya que esta etapa constituye un periodo crítico de adaptación, donde los patrones de vida pueden cambiar impactando la salud. Por ejemplo, el elevado nivel de estrés que manejan, producto de la demandante carga académica a la cual deben responder, podría influir en la AF (JerezMendoza y Oyarzo-Barría, 2015). Además, según el análisis de situación integral de salud (ASIS) 
de la Universidad de Costa Rica (UCR), entre un $60 \%$ a $70 \%$ de la población universitaria se cataloga como sedentaria (Oficina de Bienestar y Salud de la Universidad de Costa Rica (OBS), $\underline{2019)}$

Las personas universitarias pertenecientes al área de salud, y en particular la población estudiantil de enfermería, presentan un riesgo alto de sedentarismo e inactividad física; por ejemplo en Suramérica se ha reportado que el $72.6 \%$ de la población estudiantil de enfermería interrumpió con frecuencia o abandonaron las prácticas de AF o bien la mayoría realiza actividad física baja y moderada presentando a su vez un predominio del sobrepeso y obesidad (HuamanCarhuas y Bolaños-Sotomayor, 2020; Escobar, Mejía, y Betancur, 2017). Estas condiciones podrían estar relacionadas a situaciones propias del estilo de vida universitario, como lo son el número de horas sentados en las aulas y el tiempo dedicado a los estudios (Huaman-Carhuas y Bolaños-Sotomayor, 2020).

Existen diferentes aspectos que intervienen de manera positiva o negativa en el nivel de AF que presenta la población estudiantil universitaria, por lo que conocer estos aspectos es fundamental para promover la AF y por ende mejorar la salud en esta población. Según el Modelo de Promoción de la Salud, existen variables merecedoras de atención que teóricamente tienen vínculo con la adopción de conductas promotoras de salud, como lo son la percepción de barreras y beneficios de la acción y la autoeficacia (Pender, Murdaugh, y Parsons, 2014).

Los beneficios son resultados positivos anticipados que se producirán como expresión de la conducta de salud (Aristizábal, Blanco, Sánchez, y Ostiguín, 2011). Las barreras son apreciaciones negativas o desventajas de la propia persona que pueden obstaculizar un compromiso con la acción, la mediación de la conducta y la conducta real y se pueden dividir en internas como el cansancio, no saber cómo hacerlo y la pereza; externas como no tener con quien hacerlo, la falta de facilidades y no tener tiempo (Izquierdo, Hernández, y Urrutia, 2017; Aristizábal et al., 2011). Y la autoeficacia representa la percepción de competencia de uno mismo para ejecutar una conducta; la teoría sugiere que a mayor autoeficacia se perciben menos barreras y hay mayor posibilidad de realizar la conducta, por lo que no se puede desligar el pensamiento de la acción, puesto que estos marcan la conducta (Aristizábal et al., 2011).

En estudiantes universitarios y de enfermería se ha reportado que la autoeficacia para superar las barreras relacionadas con la AF tiene una asociación con la AF, especialmente la vigorosa, y que ha demostrado ser un predictor importante para la misma (Liu y Dai, 2017; Blake, Stanulewicz, y Mcgill, 2017; Seo, 2019).

Con respecto a las barreras percibidas en jóvenes universitarios para realizar AF sobresalen la percepción de falta de tiempo, la falta de compañía o apoyo social, el cansancio y 
la falta de voluntad para iniciar o mantenerse en alguna AF (Rubio y Varela, 2016; RamírezVélez, Triana-Reina, Carrillo y Ramos-Sepúlveda, 2016). En estudiantes universitarios del área de salud, incluyendo estudiantes de enfermería, que eran inactivos físicamente, las barreras principales fueron las limitaciones de tiempo, falta de acceso a lugares para realizar deporte, tener otras prioridades, ausencia de otras personas que los apoyen, horarios inconvenientes (Awadalla et al., 2014; Blake, Stanulewicz y Mcgill, 2017).

En cuanto a los beneficios específicos de la AF en estudiantes universitarios de enfermería y medicina, aquellos que han sido más percibidos están relacionados a la salud (Blake et al., 2017). Y siguiendo el modelo transteórico, se evidenció que los beneficios percibidos tenían un efecto indirecto en la AF por medio de los estadios de cambio de comportamiento los cuales a su vez tenían efecto en los procesos de cambio (Liu, Kueh, Arifin, Kim, y Kuan, 2018).

Al analizar las interacciones entre la autoeficacia y la percepción de barreras y beneficios para la actividad física en estudiantes de salud, la autoeficacia mediaba parcialmente la relación entre las barreras para la AF y las intenciones de comportamiento en salud y completamente la asociación entre barreras para la AF en general y AF de moderada a vigorosa (Olutende, Kweyu, y Wekesa, 2017). Interesantemente la población estudiantil de enfermería eran menos activos que los de medicina y percibían menos beneficios y más barreras para el ejercicio (Blake et al., 2017).

A pesar de que las investigaciones internacionales son amplias alrededor de este tema, en estudiantes universitarios de enfermería costarricenses no se tiene conocimiento de cómo se comportan las relaciones entre estas variables, por lo que el objetivo del estudio fue determinar la relación entre la autoeficacia, y la percepción de beneficios y barreras de la AF en estudiantes de enfermería. La detección de estas asociaciones podría servir para la creación de intervenciones personalizadas y eficientes, que se ajusten a las necesidades y características de cada una de las diferentes poblaciones que conforman la comunidad universitaria.

\section{METODOLOGÍA}

\section{Diseño y participantes}

La investigación se abordó desde el diseño de estudio de caso grupal, correlacional y descriptivo. Al ser un estudio de caso grupal y tener características particulares, se decidió trabajar con una muestra censal conformada por 42 estudiantes de segundo año de la carrera de Licenciatura en Enfermería de la UCR. 
Los criterios de inclusión eran: hombres o mujeres entre 18 y 25 años, ser estudiantes activos de la carrera de Enfermería, que estuvieran cursando su segundo año de carrera y al menos haber matriculado uno de los cursos correspondientes al I ciclo de segundo año de carrera. Dentro de los criterios de exclusión se encontraban estudiantes que tuvieran algún impedimento o alguna discapacidad para realizar AF (contraindicación médica para realizar AF por una patología crónica o algún cuadro agudo).

El caso de la población estudiantil de enfermería de $2^{\text {do }}$ año de la UCR es particular ya que tienen una alta demanda académica de cursos propios de carrera (Desarrollo Socio-histórico y Epistemológico, Investigación Cuantitativa) y cursos de servicio de otras escuelas (Fundamentos de Crecimiento y Desarrollo Humano, Fundamentos de Bioestadística, Fundamentos de Microbiología y Parasitología, y Principios de Fisiología Humana). Así mismo este nivel es el último antes de ingresar a los módulos de enfermería (cursos con alto creditaje, que incluyen campos clínicos y todas las clases teóricas necesarias para la práctica, demandan alrededor de 30 horas presenciales a la semana), lo que hace que las variables que podrían asociarse con la realización de AF no se repitan en las generaciones que cursan otros años de la maya curricular.

\section{Instrumentos}

Para medir las variables de interés, se utilizaron los siguientes cuestionarios autoadministrados:

Datos sociodemográficos: Se recolectaron por medio de un cuestionario de elaboración propia, el cual contenía aspectos como: edad, sexo, zona de procedencia y residencia, situación académica y laboral.

Percepción de beneficios y Barreras: Se utilizó la Escala de Beneficios y Barreras para el ejercicio (EBBE), versión en español (Pender, Noble, Sechrist, 1987; Enríquez-Reyna et al., 2017). Esta escala consta de 43 ítems que forman dos subescalas: subescala de beneficios (29 ítems) dentro de los que se incluyen la mejora de la salud mental, disminución del estrés, promoción a la socialización, mejora del bienestar; y la subescala de barreras (14 ítems) que contempla barreras como la falta de tiempo, la falta de voluntad y energía, así como el esfuerzo que implica, además de los lugares y costo económico para realizar actividad física (Pender et al., 1987). Los ítems se evalúan mediante una escala Likert de 4 puntos que va desde completamente de acuerdo a completamente en desacuerdo (la subescala de barreras tiene calificación inversa). La escala se puede calificar en su totalidad, donde a mayor puntaje más positivamente la persona percibe el ejercicio, o bien por las subescalas donde a mayor puntaje mayor percepción de los beneficios y menor es la percepción de las barreras por la población 
(Pender, et al 1987; Enríquez-Reyna et al., 2017). La confiabilidad de la escala de beneficios es de .95 y la de barreras .71, y muestra adecuada validez de constructo y discriminante (Becerra y Díaz, 2008; Enríquez-Reyna et al., 2017).

Autoeficacia: Se utilizó la Escala de Autoeficacia para la AF, la cual cuenta con 27 ítems y define tres factores conductuales para la práctica de la AF: Ejercicio físico programado (15 ítems), AF realizada en las actividades cotidianas (9 ítems), y Caminar (3 ítems) (Fernández, Medina, Herrera, Rueda, Fernández, 2011). Se evalúa por una escala tipo Likert donde se valora la autoeficacia en términos de confianza para realizar una conducta que va de 0 a 10 puntos, en la que 0 es "nada capaz" y 10 "muy capaz, cuanto mayor es la puntuación, mayor es la autoeficacia. La puntuación puede ser por subescala o la suma de las tres subescalas para obtener una puntuación global de autoeficacia para la AF (Fernández et al., 2012). Para la correcta validación de esta escala se realizó un proceso que constó de tres fases, en la primera fase la escala con 34 ítems fue revisada por expertos en el área de la investigación en la autoeficacia y las conductas de salud, las cuales evaluaron la pertinencia, claridad, precisión y suficiencia de: a) los dominios de conducta en relación a los objetivos de la escala y b) cada uno de los ítems en relación a los dominios de conducta lo cual tuvo como resultado que los factores sean considerados como pertinentes, claros, precisos y suficientes concluyendo con modificaciones una escala de 39 ítems (Fernández et al., 2011). Esta escala tiene una confiabilidad alta en todas las subescalas $(\alpha \geq 0,90$ ). El alfa de Cronbach para la escala total fue de 0.96 (subescala autoeficacia para el ejercicio físico programado 0.95 , subescala autoeficacia para actividades de la vida diaria 0.90, y subescala autoeficacia para caminar 0.91) (Fernández et al., 2011).

\section{Procedimientos}

Después de la aprobación de la investigación por parte del Comité Ético Científico de la UCR, se procedió a reclutar a los participantes mediante visitas a algunas clases de los cursos mencionados anteriormente para identificar a las personas elegibles con disposición de participar y hacer el proceso de consentimiento informado y recolección de los datos. A aquellos y aquellas estudiantes elegibles se les proporcionaron los cuestionarios en papel para que los completaran en el momento. La población estudiantil fue identificada por un código numérico y no por su nombre o alguna otra información personal, de manera que se aseguró la confidencialidad de los datos. Se hizo énfasis en que estos datos de forma individual no serían compartidos con los profesores del curso. 


\section{Análisis de datos}

Los datos se analizaron por medio de estadística descriptiva (frecuencias, medidas de tendencia central y dispersión) y después de evaluar los supuestos estadísticos (los cuales se cumplieron) se realizaron correlaciones de Pearson. Con los datos perdidos ( $<10 \%$ en todos los instrumentos) se realizó una sustitución por la media individual (Pender, et al., 1987; Cuesta, Fonseca-Pedrero, Vallejo, y Muñiz, 2013). Se utilizó también el alfa de Cronbach como una medida de consistencia interna de las escalas utilizadas. Para el análisis de los datos se utilizó el programa SPSS versión 25.

\section{RESULTADOS}

En los participantes predominó el sexo femenino con un rango de edad entre los 18-19 años, con al menos dos años de estar en la universidad y la mayoría provenía del Gran Área Metropolitana y viven con al menos 2 o 3 personas y por lo general conviven con su madre (Ver Tabla 1).

\section{Tabla 1}

Características sociodemográficas de la población participante $(n=42)$

\begin{tabular}{ccc}
\hline $\begin{array}{c}\text { Característica } \\
\text { Sociodemográfica }\end{array}$ & Frecuencia (n) & Porcentaje \% \\
\hline Sexo & 7 & $16.7 \%$ \\
Mujer & 35 & $83.3 \%$ \\
Rango de edad en años & & \\
cumplidos & 25 & $59,5 \%$ \\
$18-19$ & 12 & $28,6 \%$ \\
$20-21$ & 3 & $7,2 \%$ \\
$22-23$ & 2 & $4,8 \%$ \\
$24-25$ & & \\
2 & 31 & $73.7 \%$ \\
3 & 6 & $14.3 \%$ \\
4 o más & 5 & \\
Años en la universidad & & \\
Cambio de residencia & & $33.3 \%$ \\
durante el periodo lectivo & 14 & $66.7 \%$ \\
Si & 28 & \\
No & & $9.5 \%$ \\
Si & 4 & $2.4 \%$ \\
No & 37 & \\
No responde & 1 &
\end{tabular}

Fuente: elaboración propia con datos del cuestionario de datos sociodemográficos 


\section{Autoeficacia para la Actividad física}

En la tabla 2 se presentan los resultados de la Escala de Autoeficacia para la AF, donde la categoría caminar fue la que presentó levemente un mayor nivel de autoeficacia, seguidamente autoeficacia para realizar actividades de la vida diaria y por último autoeficacia para el ejercicio físico programado.

\section{Tabla 2}

Niveles de autoeficacia según el tipo de actividad física $(n=42)$

\begin{tabular}{|c|c|c|c|c|c|}
\hline $\begin{array}{l}\text { Autoeficacia } \\
\text { (AE) }\end{array}$ & $\begin{array}{c}\text { Promedio } \\
\text { estandarizado } \\
(0-10)\end{array}$ & $\begin{array}{c}\text { Promedio } \\
\text { No } \\
\text { estandarizado }\end{array}$ & $\begin{array}{c}\text { Desviación } \\
\text { Estándar }\end{array}$ & $\begin{array}{l}\text { Rango } \\
\text { posible }\end{array}$ & $\begin{array}{c}\text { Rango } \\
\text { obtenido }\end{array}$ \\
\hline Caminar & 7.44 & 22.31 & 7.93 & $0-30$ & $0-30$ \\
\hline $\begin{array}{l}\text { Actividades de } \\
\text { la vida diaria }\end{array}$ & 6.82 & 61.36 & 15.65 & $0-90$ & $22-90$ \\
\hline $\begin{array}{l}\text { Ejercicio físico } \\
\text { programado }\end{array}$ & 6.36 & 95.38 & 28.54 & $0-150$ & $43-149$ \\
\hline $\begin{array}{c}\text { Actividad física } \\
\text { total }\end{array}$ & 6.87 & 179.05 & 43.79 & $0-270$ & $87-269$ \\
\hline
\end{tabular}

Al analizar los ítems individuales de la escala de autoeficacia para la AF, la población estudiantil evidenció mayor nivel de autoeficacia para la AF en aquellos relacionados con caminar todos los días 60 minutos, realizar AF en la vida diaria, aunque estén de vacaciones y aunque suponga perder más tiempo y practicar ejercicio físico programado tres o más veces por semana durante al menos 20 minutos, aunque esto implique un cambio en la forma de vivir. En cuanto a los ítems con menor nivel de autoeficacia están practicar ejercicio físico programado tres o más veces por semana durante al menos 20 minutos, aunque tengan problemas en el estudio y aunque tengan mucho estudio, asimismo el realizar AF en la vida diaria aunque no tenga tiempo.

El alfa de Cronbach para la escala total de autoeficacia para la actividad física en este estudio fue de 0.92 (subescala autoeficacia para el ejercicio físico programado 0.90 , subescala autoeficacia para actividades de la vida diaria 0.80 , y subescala autoeficacia para caminar 0.91 ). 


\section{Beneficios y barreras percibidas del ejercicio}

Con respecto a los beneficios y barreras percibidas del ejercicio se obtuvo como resultado que la mayoría de personas participantes perciben más beneficios que barreras sobre el ejercicio (Ver tabla 3).

\section{Tabla 3}

Percepción de Beneficios y Barreras del Ejercicio ( $n=42)$

\begin{tabular}{lcccc}
\hline \multicolumn{1}{c}{ Variable } & Promedio & $\begin{array}{c}\text { Desviación } \\
\text { Estándar }\end{array}$ & $\begin{array}{c}\text { Rango } \\
\text { posible }\end{array}$ & $\begin{array}{c}\text { Rango } \\
\text { obtenido }\end{array}$ \\
\hline Barreras & 28.76 & 7.45 & $0-42$ & $9-42$ \\
Beneficios & 74.80 & 10.08 & $0-87$ & $48-87$
\end{tabular}

Fuente: Elaboración propia con datos del cuestionario de Escala de Beneficios/Barreras para el Ejercicio (EBBE)

Al analizar los ítems individuales de la escala de beneficios y barreras para el ejercicio y tomando como referencia únicamente las respuestas brindadas en las categorías de "Completamente de acuerdo" y "De acuerdo", el 100\% de las personas percibe como beneficios aquellos relacionados con el disfrute, disminución del estrés y tensión, mejora de la salud mental, condición física, funcionamiento general del cuerpo y apariencia física. Por el contrario, los beneficios que las personas menos seleccionaron (76\%) fueron tener contacto con amistades, conocer personas nuevas y el mejoramiento de la percepción que otros tienen de mí. Por su parte $42 \%$ de las personas percibieron como barreras las relacionadas con la cantidad de tiempo que toma, cansancio y fatiga provocado por el ejercicio, y las barreras menos seleccionadas $(9.5 \%)$ fueron vergüenza por hacer ejercicio o atuendos graciosos.

El alfa de cronbach para la escala de barreras para el ejercicio fue de 0.87 y la escala de beneficios para el ejercicio fue 0.93 .

\section{Asociaciones entre la autoeficacia y la percepción de barreras y beneficios}

Se identificaron correlaciones significativas positivas entre: 1) la autoeficacia total para la AF y la percepción de beneficios del ejercicio $(r=0.31, p<0,05)$, a mayor nivel de autoeficacia para la $A F$, mayor percepción de los beneficios que presenta el ejercicio físico en las personas; 2 ) los beneficios para el ejercicio y la autoeficacia para caminar todos los días $(r=0.30, p<0,05)$, a mayor percepción de los beneficios del ejercicio, mayor nivel autoeficacia para caminar todos los días; 3) la percepción de barreras para el ejercicio y la autoeficacia para el ejercicio físico programado 
$(r=0.32, p<0,05)$, a mayor percepción de las barreras, mayor nivel de autoeficacia para el ejercicio físico programado (ver Tabla 4)

Tabla 4.

Correlaciones entre las variables principales $(n=42)$

\begin{tabular}{lcc}
\hline \multicolumn{1}{c}{ Variable } & Barreras para el Ejercicio & $\begin{array}{c}\text { Beneficios del } \\
\text { Ejercicio }\end{array}$ \\
\hline 1. AE para la actividad física (Total) & 0.17 & $0.31^{*}$ \\
2. AE Ejercicio físico programado & $0.32^{*}$ & 0.27 \\
3. AE Actividades de la vida diaria & -0.11 & 0.17 \\
4. AE Caminar & 0.03 & $0.36^{*}$ \\
\hline
\end{tabular}

${ }^{*} p<0,05 \quad{ }^{* *} p<0,01$

Fuente: Elaboración propia con los datos de los cuestionarios de Escala de Autoeficacia para la Actividad Física y Escala de Beneficios/Barreras para el Ejercicio (EBBE)

\section{DISCUSIÓN}

El objetivo del estudio fue analizar las relaciones entre la autoeficacia y la percepción de beneficios y barreras de la AF en estudiantes de enfermería. Se encontraron asociaciones positivas entre percepción de los beneficios y la autoeficacia total para la AF, así como entre percepción de barreras y autoeficacia para realizar ejercicio programado, ambas asociaciones con un coeficiente mediano. Lo anterior coincide con los hallazgos Blake et al. (2017) quienes encontraron una asociación positiva significativa grande $(r=0.58, p<0.01)$ entre la autoeficacia, la percepción de barreras y beneficios en estudiantes de enfermería y medicina. Al colocar estas variables en un modelo predictivo para la actividad física en estos estudiantes se obtuvo que solamente la autoeficacia fue un predictor significativo. En otras poblaciones, como en adultos medios y mayores, estas asociaciones también se han encontrado (Enríquez et al., 2017; Ayotte, Margrett, y Hicks-Patrick, 2010)

La percepción anticipada de resultados positivos que producirá eventualmente una conducta (autoeficacia), tiene como base las memorias personales que se producen con la experiencia previa o el aprendizaje vicario, de forma que las personas van a invertir el tiempo y recursos en aquellas actividades que presentan una alta probabilidad de incrementar sus experiencias con resultados positivos (Enríquez-Reyna et al., 2017). La adecuada percepción de beneficios podría ser un mecanismo que eleva la sensación de capacidad para realizar ejercicio en general y caminar. Por su parte, aquellos y aquellas estudiantes que son capaces de identificar las barreras son los que se sienten más capaces de realizar ejercicio programado; esta 
identificación les podría ayudar a manejar o superar estas barreras para efectivamente realizar actividad física con mayor nivel de autoeficacia.

Los resultados descriptivos respaldan que la población estudiantil tiene niveles de autoeficacia total para la AF con tendencia a puntajes altos y que identifican con mayor puntualidad los beneficios que trae consigo la práctica de la AF, y en menor medida aquellas barreras que pueden interferir. Este resultado se compara con un estudio realizado con universitarios donde se encontró que la población tiene un nivel moderado de autoeficacia para la $A F$, lo cual valida el rol de la autoeficacia, en la adopción y mantenimiento de la AF como una conducta de salud, de manera que a mayor autoeficacia la población estudiantil percibe mayor capacidad de realizar correctamente planes de acción y afrontamiento en relación a la AF, ya que confían en sus capacidades para conocer cómo, cuándo, y dónde realizar AF así como también confían en sus capacidades para lidiar con los obstáculos que puedan encontrar (Barberán y Roa, 2016). También se ha determinado que un mayor nivel de AF se predice significativamente por un mayor nivel de autoeficacia para la práctica de AF (Mella, 2020).

El rol de la autoeficacia en la adopción y mantenimiento de la AF como una conducta de salud, es esencial ya que a mayor autoeficacia la población estudiantil percibe mayor capacidad de realizar correctamente planes de acción y afrontamiento en relación a la AF, ya que confían en sus capacidades para conocer cómo, cuándo, y dónde realizar AF así como también confían en sus capacidades para lidiar con los obstáculos que puedan encontrar (Barberán y Roa, 2016).

Caminar es la AF que las personas sienten más capacidad para realizar, lo cual es importante y coherente ya que representa un bajo costo económico y considerando el poco tiempo libre del estudiantado, junto con el cansancio que genera la carga académica, caminar resulta una de las actividades físicas que mejor se ajusta a las particularidades de esta población (Rodríguez, 2011). Por el contrario, la autoeficacia para la AF programada, aquella que se planifica, como ir al gimnasio, o realizar algún otro tipo de entrenamiento, es la que menores niveles de autoeficacia presenta. Una de las posibles razones podría ser el tiempo que demanda el ocuparse de las responsabilidades académicas y no contar con el tiempo suficiente. La carga académica podría ser entonces un factor determinante que limite la autoeficacia para realizar AF programada (Molano-Tobar, Vélez-Tobar, y Rojas-Galvis, 2019).

En lo referente a los beneficios y barreras percibidas de la AF, se evidenció que la mayoría del estudiantado percibe más beneficios que barreras. Los resultados concuerdan con los hallazgos encontrados por Cruz et al. (2017) quienes afirman que los jóvenes universitarios perciben más beneficios e influencia de amigos hacia la práctica de AF que los jóvenes de secundaria. Al respecto Fernández y Ropero $(\underline{2015})$ en su estudio acerca de la percepción de 
beneficios, barreras y nivel de AF en estudiantes universitarios colombianos, demostraron que la población estudiantil tiene una alta percepción sobre los beneficios del ejercicio y una mediana percepción de las barreras del ejercicio.

Los beneficios percibidos con mayor puntaje, por parte de la población estudiantil, están relacionados con la salud mental, coincidiendo esto con un estudio que afirma que, si la AF se realizaba en el ámbito del tiempo libre, en un individuo "suficientemente activo", disminuye el riesgo de padecer patologías de salud mental en un $56 \%$ con respecto a un individuo "insuficientemente activo" (Rodríguez et al., 2015).

En cuanto a los beneficios con menor percepción, la socialización que se fomenta mediante la $A F$ no es un beneficio percibido por la población estudiantil de enfermería de la UCR. Esto puede estar relacionado con la demanda de tiempo que genera la malla curricular tanto para asistir a clases presenciales como para cumplir con las asignaciones y estudios fuera de clase, lo que hace que el tiempo destinado para la práctica de AF no se preste tanto para socializar. Esta idea va en línea con El-Gilany, Badawi, El-Khawaga, y Awadalla (2011), donde observaron que la percepción de la socialización como beneficio de la AF obtuvo el penúltimo lugar en relevancia para la población que estudiaron.

Entre las principales barreras para el ejercicio encontradas en este estudio se resalta que el tiempo es un factor clave, así como también el esfuerzo implicado al realizar AF. Rubio y Varela (2016) y Castañeda, Zagalaz, Arufe, y Campos-Mesa (2018) coinciden con que las barreras a las que se enfrentan los universitarios son: la falta de tiempo, la falta de energía o ganas y la falta de voluntad o interés. Además, Mella (2020) evidenció que específicamente, a mayor falta de tiempo y falta de voluntad menor nivel de AF y, a mayor falta de habilidad mayor nivel de AF. En relación con la falta de tiempo Huaman-Carhuas y Bolaños-Sotomayor (2020) señalan que la población estudiantil universitaria incrementa el número de horas que permanece sentado en las aulas, así como el tiempo dedicado al estudio con respecto al alumno de educación secundaria, haciéndose cada vez más sedentario, es decir, que dispone de menos tiempo libre para realizar actividad física, también concluyeron que conforme avanzan los años y se desarrollan nuevas tecnologías diversas, la realización de actividad física disminuye sobre todo en la población joven.

Sumado a lo anterior, relacionado específicamente a la barrera de fatiga provocada por el ejercicio, se encontraron resultados similares en otro estudio con población universitaria ya que un $59.8 \%$ de la población estudiantil universitaria estuvieron de acuerdo con esta afirmación (Arboleda, Arango, y Feito, 2016). Respecto de la percepción de barreras, este estudio confirma 
su influencia en la práctica de AF, mostrando que, a mayor falta de tiempo y falta de voluntad, menor nivel de AF, y a mayor falta de habilidad, mayor nivel de AF

Las barreras antes mencionadas pueden deberse a las características que presenta la población estudiantil, donde aspectos como: la carga académica, los tiempos de clases, las demandas requeridas fuera del tiempo de clases, el cansancio durante la semana y la falta de sueño, promuevan que el tiempo que no es destinado a actividades académicas ni sociales sea invertido en descansar, dejando de lado la práctica de AF (Rubio y Varela, 2016).

La principal limitación de la presente investigación fue la no evaluación del nivel de actividad física, lo cual impidió el análisis de las relaciones de la autoeficacia, percepción de beneficios y barreras con el nivel de actividad física de la población estudiantil de enfermería. Para futuras investigaciones relacionadas con la temática y la población se recomienda la medición del nivel de AF con instrumentos, de preferencia objetivos, para así poder establecer relaciones predictivas o modelos que expliquen la adopción o no de este comportamiento de salud. Además, tomar una muestra representativa y estratificada de toda la población estudiantil que conforman la escuela de enfermería para poder valorar las diferencias que se puedan presentar en las variables relacionadas con la AF según el año de carrera que curse la población que se estudie.

A pesar de esto existen fortalezas en este estudio ya que marca un punto de referencia para replicar en el futuro este tipo de investigaciones en la población durante toda la carrera de enfermería, con el fin de identificar y profundizar en los elementos que podrían estar involucrados en la adopción de conductas saludables como lo es la autoeficacia, y la percepción de beneficios y barreras. A partir de los resultados obtenidos se podrían justificar programas que busquen promover un mayor nivel de autoeficacia para la práctica de la AF en la población universitaria así como detectar las barreras buscar disminuirlas o bien sobrellevarlas e incentivar y promover los beneficios de la AF. Otro punto fuerte de esta investigación es la posibilidad de generar futuras hipótesis que brinden una base para estudios más amplias de este fenómeno en la población de estudiantes de enfermería y del área de la salud en Costa Rica. Se recomienda diseñar estrategias que ayuden a las personas a realizar las pautas recomendadas de actividad física desde los diferentes sectores sociales, políticos y educativos, de esta manera se ayudaría a la población universitaria a que se mantenga físicamente activa en esta etapa y no disminuya su nivel de actividad física debido al exceso de trabajo que implican los estudios, y así disminuir las repercusiones en la vida adulta (García, Caracuel, y Ceballos, 2014). 


\section{CONCLUSIÓN}

En la población estudiantil de enfermería costarricense de segundo año de carrera de la UCR, la autoeficacia para la AF en general tuvo un nivel con tendencia al alta, siendo caminar el tipo de AF que se sentían más capaces de realizar. Perciben a su vez más beneficios que barreras de la $A F$, siendo los beneficios relacionados a la salud mental y física y las barreras de falta de tiempo y fatiga las que más identifican. Se concluye en este caso grupal de estudiantes de enfermería que el percibir más barreras está asociado a un aumento en la autoeficacia para el ejercicio programado, así como que el percibir más beneficios está asociado a un aumento en la autoeficacia general para la AF y caminar.

A pesar de que los profesionales en enfermería cuentan con conocimientos acerca del adecuado mantenimiento de la salud por medio de la actividad física, es importante buscar mecanismos que colaboren con la adopción y el mantenimiento de estos comportamientos desde que están en formación. Investigaciones como esta aportan evidencia para facilitar en un futuro el abordaje de la temática utilizando diferentes estrategias más personalizadas con el fin de fomentar la AF en el estudiantado y por ende mejorar su salud y bienestar para que a la vez estos sirvan de ejemplo a las personas que cuidan.

\section{REFERENCIAS}

Arboleda, V., Arango, E., y Feito, Y. (2016). Actividad Física y Percepción de Beneficios y Barreras en una Universidad Colombiana. Retos: Nuevas Tendencias en Educación Física, Deporte $\quad y \quad$ Recreación, (30), 15-19. Recuperado de https://www.redalyc.org/pdf/3457/345744747003.pdf

Aristizábal, G., Blanco, D., Sánchez, A., y Ostiguín, R. (2011). El modelo de promoción de la salud de Nola Pender: Una reflexión en torno a su comprensión. Enfermería universitaria, 8(4),16-23. Recuperado de http://www.scielo.org.mx/scielo.php?script=sci arttext\&pid=S1665$70632011000400003 \& \operatorname{lng}=e s \& t \operatorname{lng}=e s$

Awadalla, N. J., Aboelyazed, A. E., Hassanein, M. A., Khalil, S. N., Aftab, R., Gaballa, I. I., y Mahfouz, A. A. (2014). Assessment of physical inactivity and perceived barriers to physical activity among health college students, south-western Saudi Arabia. Eastern Mediterranean health journal $=$ La revue de sante de la Mediterranee orientale, 20(10), 596-604. doi: https://doi.org/10.26719/2014.20.10.596 
Ayotte, B., Margrett, J., y Hicks-Patrick, J. (2010). Physical Activity in Middle-aged and YoungOld Adults. The Roles of Self-Efficacy, Barriers, Outcome Expectancies, Self-regulatory Behaviors and Social Support. Journal of Health Psychology, 15(2), 173-85. Recuperado de

https://www.researchgate.net/publication/41808296 Physical Activity in Middleaged an d Youngold Adults The Roles of Selfefficacy Barriers Outcome Expectancies Selfregulatory Behaviors and Social Support

Barberán, C., y Roa, J. (2016). Actividad física en universitarios y su relación con autoeficacia y percepción de riesgo (Tesis de grado). Pontificia Universidad Javeriana Cali, Colombia. Recuperado de http://vitela.javerianacali.edu.co/handle/11522/4471

Becerra, M., y Diaz, L. (2008). Niveles de actividad física beneficios barreras y autoeficacia en un grupo de empleados oficiales. Avances en Enfermería, 26(2), 43-50. Recuperado de https://revistas.unal.edu.co/index.php/avenferm/article/view/12897/13498

Blake, H., Stanulewicz, N., y Mcgill, F. (2017). Predictors of physical activity and barriers to exercise in nursing and medical students. Journal of Advanced Nursing, 73(4), 917-929. doi: https://doi.org/10.1111/jan.13181

Castañeda, C., Zagalaz, M., Arufe, V., y Campos-Mesa, M. (2018). Motivos Hacia La Práctica de Actividad Física de los Estudiantes Universitarios. Revista Iberoamericana de Psicología del Ejercicio $y$ el Deporte, 13(1), 79-89. Recuperado de http://www.redalyc.org/articulo.oa?id=311153534008

Cruz-Castruita, R. M., González, M. J., Raimundi, M. J., y Enríquez-Reyna, M. C. (2017). Beneficios, barreras y apoyo social para el ejercicio en universitarios: diferencias por grado escolar. Revista de Psicología del Deporte, 26(2), 165-171. Recuperado de https://ri.conicet.gov.ar/handle/11336/72592

Cuesta, M., Fonseca-Pedrero, E., Vallejo, G., y Muñiz, J. (2013). Datos perdidos y propiedades psicométricas en los tests de personalidad. Anales de Psicología, 29(1), 285-292. doi: https://dx.doi.org/10.6018/analesps.29.1.137901

El-Gilany, A.H., Badawi, K., El-Khawaga, G., y Awadalla, N. (2011). Physical activity profile of students in Mansoura University, Egypt. EMHJ - Eastern Mediterranean Health Journal, 17(8), 694-702. Recuperado de https://apps.who.int/iris/handle/10665/118286

Enríquez-Reyna, M., Cruz-Castruita, R., Ceballos-Gurrola, O., García-Cadena, C., HernándezCortés, P., y Guevara-Valtier, M. (2017). Propiedades psicométricas a Escala de Beneficios/Barreras para o Ejercicio en ancianas mexicanas. Revista Latino-Americana de 
Enfermagem, 25, e2902. Recuperado de https://www.scielo.br/pdf/rlae/v25/es 0104-1169rlae-25-e2902.pdf

Escobar, M., Mejía, A., y Betancur, S. (2017). Cambios en el autocuidado de estudiantes colombianos de enfermería, durante la formación profesional: 2015. Hacia la Promoción de la Salud, 22(1), 27-42. Recuperado https://bit.ly/3wUVc2e

Fernández, C., y Ropero, F. (2015). Percepción de beneficios, barreras y nivel de actividad física de estudiantes universitarios. Investigaciones Andina, 17(31), 1391-1406. Recuperado de https://www.redalyc.org/pdf/2390/239040814009.pdf

Fernández, T., Medina, S., Herrera, I, Rueda, S., Fernández, A. (2011). Construcción y validación de una escala de autoeficacia para la actividad física. Revista Española de Salud Pública, 85(4), 405-417. Recuperado de https://www.redalyc.org/pdf/170/17019926009.pdf

Fernández, T., Medina, S., Herrera, I., Rueda, S., Ordóñez, J., León-Rubio, J., Cantero, F. (2012). Guía de uso de la escala de autoeficacia para la actividad física. Recuperado de https://www.researchgate.net/publication/257304109 Guia de uso de la escala de aut oeficacia para la actividad f'isica/link/54871bb50cf2ef34478eb39f/download

García, J., Caracuel, J., y Ceballos, O. (2014). Motivación y ejercicio físico deportivo: Una añeja relación. Revista Internacional de Ciencias Sociales y Humanidades, SOCIOTAM, 24(1), 71-88. Recuperado de https://www.redalyc.org/articulo.oa?id=654/65452570005

Huaman-Carhuas, L. y Bolaños-Sotomayor, N. (2020). Sobrepeso, obesidad y actividad física en estudiantes de enfermería pregrado de una universidad privada 2017. Enfermería Nefrológica, 23(2), 184-190. doi: https://doi.org/10.37551/s2254-28842020018

Izquierdo, L., Hernández, M., y Urrutia, O. (2017). Barreras para la práctica de actividades físicas en estudiantes de la Escuela Latinoamericana de Medicina. Panorama Cuba y Salud, 12(S1), 6-8. Recuperado de https://www.medigraphic.com/pdfs/cubaysalud/pcs2017/pcss171b.pdf

Jerez-Mendoza, M., y Oyarzo-Barría, C. (2015). Estrés académico en estudiantes del Departamento de Salud de la Universidad de Los Lagos Osorno. Revista chilena de neuropsiquiatría, 53(3), 149-157. doi: https://doi.org/10.4067/S0717-92272015000300002

Liu, H., y Dai, X. (2017). Correlation between physical activity and self-efficacy in Chinese university students. Revista de psicología del deporte, 26(4), 110-114. Recuperado de https://ddd.uab.cat/pub/revpsidep/revpsidep a2017v26sup4/revpsidep a2017v26n6p110. pdf 
Liu, K. T., Kueh, Y. C., Arifin, W. N., Kim, Y., y Kuan, G. (2018). Application of transtheoretical model on behavioral changes, and amount of physical activity among university's students. Frontiers in psychology, 9, 2402. doi: https://doi.org/10.3389/fpsyg.2018.02402

Mella, J. A. (2020). Motivación, autoconcepto, autoeficacia, bienestar y barreras hacia la actividad física en universitarios (Tesis de Maestría). Universidad de Concepción, Colombia. Recuperado de http://repositorio.udec.cl/jspui/handle/11594/509

Molano-Tobar, J., Vélez-Tobar, A., y Rojas-Galvis, A. (2019). Actividad física y su relación con la carga académica de estudiantes universitarios. Hacia la Promoción de la Salud, 24(1), 112120. Recuperado de http://promocionsalud.ucaldas.edu.co/downloads/Revista24(1) 10.pdf

Oficina de Bienestar y Salud de la Universidad de Costa Rica (OBS). (2019). Actividad Física y Recreación. Recuperado de https://obs.ucr.ac.cr/prosalud/temas/actividad-fisica-yrecreacion/

Olutende, O. M., Kweyu, I. W., y Wekesa, J. S. (2017). Health Behaviour Intentions and Barriers to Physical Activity among Health Science Students: Exploring General Self Efficacy as a Mediator. Journal of Physical Activity Research, 2(2), 101-106. doi: https://doi.org/10.12691/jpar-2-2-6

Organización Mundial de la Salud [OMS]. (2018). Programas y Proyectos: Estrategia mundial sobre régimen alimentario, actividad física y salud. Actividad física. Recuperado de https://www.paho.org/hq/dmdocuments/2012/OMS-Estrategia-Mundial-RegimenAlimentario-2004.pdf

Pender, J., Noble, S. y Sechrist, K. (1987). Health Promotion Model Instrumentation Group Health Promotion Model - Instruments to Measure HPM Behavioral Determinants: Exercise Benefits/Barriers Scale [EBBS] (Adult Version). Recuperado de https://deepblue.lib.umich.edu/handle/2027.42/85354

Pender, N., Murdaugh, C., y Parsons, M. (2014). Health Promotion in Nursing Practice (7th ed.). Pearson.

Ramírez-Vélez, R., Triana-Reina, H., Carrillo, H., y Ramos-Sepúlveda, J. (2016). Percepción de barreras para la práctica de la actividad física y obesidad abdominal en universitarios de Colombia. Nutrición Hospitalaria, 33(6), 1317-1323. Recuperado de http://scielo.isciii.es/scielo.php?pid=S0212$16112016000600010 \&$ script $=$ sci arttext\&tlng $=p t$

Rodríguez, M. (2011). Caminar 10000 pasos al día para mantener una buena salud y calidad de vida. InterSedes: Revista de las Sedes Regionales, 12(24), 137-145. Recuperado de http://www.redalyc.org/pdf/666/66622581009.pdf 
Rodríguez-Romo, G., Barriopedro, M., Salazar, P., y Garrido-Muñoz, M. (2015). Relaciones entre Actividad Física y Salud Mental en la población adulta de Madrid. Revista de Psicología del Deporte, 24(2), 233-239. Recuperado de https://www.redalyc.org/pdf/2351/235141413005.pdf

Rubio, R., y Varela, M. (2016). Barreras percibidas en jóvenes universitarios para realizar actividad física. Revista Cubana de Salud Pública, 42(1), 61-69. Recuperado de https://www.scielosp.org/scielo.php?pid=S0864-34662016000100007\&script=sci abstract

Seo, K. (2019). Factors affecting regular physical activity in nursing students. Journal of Health Informatics and Statistics, 44(1), 92-101. doi: https://doi.org/10.21032/jhis.2019.44.1.92 